\title{
APROXIMACIÓN A LOS CONOCIMIENTOS TEÓRICOS DE ENFERMERÍA SOBRE FUNCIÓN RENAL RESIDUAL
}

\author{
Diana Catalina Isaza Moreno*, Santiago Martínez Cautiva*, Lila Quintero**
}

\section{Resumen}

La función renal residual representa para algunos enfermeros de unidades renales un término indiferente en su diaria relación con el paciente renal crónico, a pesar de que su declinación tiene un efecto directo sobre la morbimortalidad. Objetivo: describir los conocimientos teóricos sobre la FRR, del enfermero que labora en programas de diálisis. Metodología: La primera fase fue el diseño del instrumento con validación de apariencia y de contenido por cinco profesionales expertos, proponiendo un cuestionario final con cuatro dominios y 25 preguntas. En la segunda fase se realizó un estudio descriptivo de corte transversal mediante la aplicación del instrumento a once enfermeros de una UR de Bogotá. Resultados: de los 11 el 82\% (9) son mujeres y 18\% (2) hombres, $64 \%$ tiene formación en posgrado y el tiempo mediano de experiencia laboral en unidad renal fue cinco años. Los que presentaron mayor número de respuestas correctas tienen edad promedio de 34,6 años y la mediana de experiencia laboral de seis años, en contraste aquellos con mayor número de respuestas incorrectas tienen cifras de 29,5 y tres años. Hubo más respuestas correctas en el dominio importancia de preservar la función renal residual y menos en los ítems que evalúan malnutrición y anemia (55 y 64\%). El dominio con menor frecuencia de respuestas acertadas fue el técnico científico (determinación de la FRR con recolección y medición de orina ínterdialítica) con 82 y $45 \%$. En el dominio de preservación de la FRR las respuestas fueron incorrectas en ultrafiltración y cambios bruscos de volemia, en 82 y $73 \%$. Conclusión: los resultados muestran la necesidad de revisar algunos conceptos teóricos y se propone desarrollar actividades académicas para fortalecerlos para contribuir a brindar un mejor cuidado de enfermería a los pacientes en terapia dialítica. Se requieren de estudios futuros para evaluar si existe relación entre el conocimiento de la FRR con la práctica que ejerce el enfermero para preservarla.

Palabras clave: función renal residual, programa de diálisis crónico, conocimiento de enfermería, conceptos teóricos sobre función renal residual.

Abreviaturas: FRR, función renal residual; UR, unidad(es) renal(es).

\section{ESTIMATE OF THE THEORETICAL KNOWLEDGE OF NURSES ON RESIDUAL RENAL FUNCTION}

\section{Abstract}

Residual renal function (RRF) is a term that makes little difference to some nursing professionals working in renal units in their daily relation with patients with chronic kidney disease although minimizing this concept has a direct effect on morbidity and mortality. Objective: to describe the theoretical knowledge nurses working in dialysis programs have on RRF. Methodology: During the first phase five expert professionals designed the

Fecha recibido: julio 28 de 2008 - Fecha aceptado: mayo 29 de 2009

* Estudiantes de especialización en Enfermería Nefrológica y Urológica, Fundación Universitaria de Ciencias de la Salud, Bogotá D.C. Colombia.
** Enfermera docente, Profesora Titular, Asesora Temática. Fundación Universitaria de Ciencias de la Salud, Bogotá DC. Colombia. 
instrument including validity of appearance and content proposing a final 4-domain, 25 questions questionnaire. In the second phase a cross sectional descriptive study was conducted applying the instrument to 11 nurses of a renal unit in Bogotá. Results: of the 11 nurses $82 \%$ (9) were females and $18 \%$ (2) males, $64 \%$ have a postgraduate degree and the median years work experience in a renal unit was five years. The average age of those achieving the greatest number of correct answers was 34.6 years and their median years work experience was six years, in contrast, the average age of those obtaining the greatest number of incorrect answers was 29.5 years and three years work experience. There were more correct answers in the domain on the importance of preserving residual renal function, and less in the items assessing malnutrition and anemia, (55\% and $64 \%$ ). The domain in which less number of correct answers was achieved was that dealing with technical scientific issues (assessing RRF by interdialysis urine collection and measurement) with $82 \%$ and $45 \%$. In the domain of RRF preservation the answers about ultrafiltration and sudden volemic changes were incorrect, in 82 and $73 \%$. Conclusion: the results demonstrate the need to review some theoretical concepts and has the purpose of developing academic activities for strengthening these concepts and contribute to offer a better nursing care to patients undergoing dialysis therapy. Future studies are required to evaluate if a relation between knowledge on RRF and nursing practices to preserve it exists.

Key words: residual renal function, chronic dialysis program, nursing professionals' ${ }^{\prime}$ knowledge, theoretical concepts on residual renal function.

\section{Introducción}

La incidencia de la enfermedad renal en Colombia se ha estimado en 12 por cada 100.000 habitantes para una población de 43 millones, afectando en especial a la población adulta. Hay un aumento anual debido a los malos hábitos y estilos de vida inapropiados de los pacientes con afecciones crónicas como hipertensión arterial y diabetes. ${ }^{1}$ Los pacientes con enfermedad renal terminal son incluidos en diálisis crónica, ya sea peritoneal o hemodiálisis, cuando la tasa de filtración glomerular está por debajo de $15 \mathrm{cc} / \mathrm{min}$, con el objeto de mejorar su pronóstico y calidad de vida; una vez iniciado el programa de diálisis a esta filtración glomerular se le llama FRR y el equipo de salud centra su atención en preservarla ya que su disminución representa un deterioro clínico progresivo.

La importancia de la FRR incide en casi todos los sistemas del organismo. Su función esencial es depurativa pues al ser continua consigue una mayor eliminación de solutos, sobre todo de mediano y alto peso molecular, que con la hemodiálisis intermitente convencional y según las características de filtración de la membrana glomerular, no son fáciles de eliminar como la â2 microglobulina. ${ }^{2}$ La FRR mantiene un mejor control hidroelectrolítico ya que el volumen de diuresis residual permite una mayor ingesta de líquidos. Así es posible mantener una dieta menos estricta facilitando un mejor control del estado nutricional, de la volemia, del peso seco y en algunos casos, de la tensión arterial. Como en el transcurso de la diálisis la principal causa de mortalidad es cardiovascular, tanto la hipertensión como la hipertrofia ventricular izquierda son importantes factores de riesgo que pueden prevenirse con el ajuste adecuado del peso seco. ${ }^{2,3}$ Otras funciones que se mantienen son las propiedades endocrinas del riñón "enfermo". La síntesis de eritropoyetina por el tejido que todavía está preservado, contribuye a un mejor control de la anemia asociada con la insuficiencia renal y por consiguiente permite disminuir las necesidades de eritropoyetina humana recombinante. La transformación de la vitamina $\mathrm{D}$ a su forma activa se realiza a nivel renal, demostrándose que los pacientes sin FRR adecuada mantienen niveles más bajos de calcitriol y menor absorción intestinal de calcio que aquellos que conservan sus riñones propios. De esta manera, la FRR también interviene, de forma secundaria, en la prevención de la osteodistrofia renal. ${ }^{2}$

La importancia clínica de la FRR se empezó a divulgar a mediados de los años 90 con el estudio de CANUSA, donde la evaluaron como factor separado y demostraron que es la persistencia de la FRR la que contribuye a la supervivencia de los pacientes en diálisis peritoneal y no la dosis 
de la diálisis peritoneal. Esto también se ha observado en pacientes en hemodiálisis. ${ }^{4}$ Preservar la FRR depende de diversos factores entre los cuales se encuentra la propia técnica de diálisis. En numerosas publicaciones se refiere que la pérdida de la FRR es más rápida en hemodiálisis comparada con la peritoneal. Esto se puede explicar por los cambios bruscos en la volemia que conducen a hipotensión, lo que a su vez produce hipoperfusión renal que conlleva daño del tejido renal remanente, es decir afecta las pocas nefronas que quedan funcionales debido al hecho que los pacientes en hemodiálisis presentan cambios bruscos de volemia, lo que no ocurre en la peritoneal continua ambulatoria. ${ }^{2,3,5}$ La técnica de hemodiálisis favorece esta pérdida debido al hecho de requerir el circuito extracorpóreo y las membranas de diálisis pueden ser estímulos potentes para la producción de citoquinas y otros mediadores inflamatorios que podrían actuar de forma deletérea sobre la FRR. La diálisis peritoneal usa como "membrana" el peritoneo que no produce este efecto adverso y es la continua ambulatoria la que preserva mejor la FRR. No ocurre lo mismo con la diálisis peritoneal intermitente automatizada y se ha descrito que son los cambios bruscos de la volemia y no la técnica en sí, los responsables de la mayor y más rápida pérdida de FRR. ${ }^{3,5}$

Aparte de la propia técnica de diálisis existen otros hechos que influyen en la pérdida de la FRR, como son el uso de fármacos neurotóxicos, sobre todo aminoglucósidos antiinflamatorios no esteroideos y los inhibidores de la enzima de conversión de angiotensina, así como el empleo de medio de contraste yodado. ${ }^{5}$ Otros factores relacionados con pérdida de FRR tienen que ver con la etiología de la enfermedad renal que motivó el ingreso a diálisis. Los pacientes con patología glomerular son los que más rápido la pierden, comparados con los que padecen una nefritis tubulointersticial o una afección vascular renal. ${ }^{5}$ El propio paciente puede ser en parte responsable de una pérdida más rápida de su FRR como sucede cuando hay altas ganancias de peso interdialítico, lo que obliga a usar grandes volúmenes durante la terapia favoreciendo cambios bruscos de volemia.

Es de vital importancia que el enfermero conozca y aplique los cuidados que ayudan a preservar la FRR, ya que parte de su pérdida se ha relacionado con la técnica y los cuidados durante la diálisis. El enfermero es quien planea, organiza, ejecuta y asiste al paciente durante la te- rapia dialítica apoyada en el tratamiento médico y nutricional. El proceso de atención de enfermería está dirigido al cuidado asistencial como al educativo, que juega un papel de motivación y regulación del autocuidado del paciente, con el fin de prevenir factores o situaciones que alteren la integridad y deterioro de su salud. La ley colombiana de enfermería 266 de 1996 y la Ley 911 de 2004 fundamentan su práctica en los conocimientos sólidos y actualizados de las ciencias biológicas, sociales y humanísticas, y en sus propias teorías y tecnologías. La Organización Panamericana de la Salud en la propuesta sobre reorientación de la educación del personal de salud, abre un espacio para la investigación sobre competencias y desempeños de estos profesionales. Propone la formación de un grupo latinoamericano que se dedique al estudio de las competencias mínimas de egreso y su correspondencia con las competencias ocupacionales. ${ }^{6,7} \mathrm{La}$ aproximación a la evaluación de competencias tiene implícita la exploración del conocimiento teórico, que si bien es elemento integrador en el saber y el hacer, no es el único eje. Este trabajo propone explorar estos elementos teóricos y abre espacios para investigaciones futuras que evalúen conocimientos prácticos, actitudes y habilidades de quienes ejercen en el campo de la salud.

\section{Métodos}

Se desarrolló en dos fases. La primera para la construcción del cuestionario y la segunda para la aplicación de éste a los profesionales de enfermería que laboran en una UR.

\section{Fase 1: diseño de cuestionario}

Objetivo: construir un instrumento para identificar los conocimientos teóricos de enfermería sobre la FRR sometido a validación de apariencia y contenido con la participación de expertos en el tema. Metodología: el grupo investigador desarrolló un primer instrumento fundamentado en la revisión bibliográfica sobre la de la FRR. Para la elaboración de preguntas se tuvo en cuenta el lenguaje claro, sencillo, sin ambigüedad ni formulación de preguntas negativas o dobles. El instrumento inicial contenía preguntas abiertas y cerradas y fue el punto de partida para ser sometido a juicio de tres expertos (un 


\section{Conocimientos de la FRR}

Cuestionario dirigido a enfermeros que laboran en UR en el programa de diálisis crónica; el objetivo es describir los conocimientos de enfermería para preservar la FRR. La participación es voluntaria y se omite la identificación de la persona.

\section{Instructivo general}

A continuación encontrará una serie de preguntas de selección múltiple y dicotómica con única respuesta verdadera. Solicitamos su colaboración para diligenciar la encuesta.

1. ¿Qué es la función renal residual (FRR) de los pacientes en diálisis crónica?

a. Función renal que mantiene el paciente con insuficiencia renal agua en programa de diálisis.

b. Reducción progresiva de la función renal del paciente con insuficiencia renal aguda en diálisis.

c. Función renal que mantiene el paciente con insuficiencia renal crónica en diálisis crónica que permite eliminar sustancias tóxicas de alto y mediano peso molecular.

d. b y c son ciertas.

2. Para determinar la FRR la recolección de orina interdialítica debe ser:
a. $200 \mathrm{cc}$.
b. $400 \mathrm{cc}$.
c. $600 \mathrm{cc}$.
d. $>1.000 \mathrm{cc}$.

\section{Para la medición de la FRR normal se tienen en cuenta los siguientes pasos, excepto: \\ a. Recolección de orina interdialítica larga. \\ b. Promedio de BUN sanguíneo pre y posdiálisis. \\ c. Tiempo de recolección en minutos. \\ d. Nitrógeno ureico urinario.}

\section{4. ¿Cuál es la importancia de preservar la FRR?}

a. Eliminar toxinas de mediano y alto peso molecular.

b. Mejorar la regulación del volumen extracelular.

c. Controlar la anemia y la osteodistrofia renal.

d. Disminuir la morbimortalidad del paciente.

e. Todas las anteriores.

\section{Responda verdadero o falso:}

La pérdida de la FRR aumenta el riesgo de presentar
a. Malnutrición ( )
b. Hipertrofia cardiaca ( )
c. Osteodistrofia renal ( )
d. Anemia ( )
e. Hipervolemia ( )

Fecha: Edad: Sexo: $\mathbf{M} \square \mathbf{F} \square$

Nivel académico: pregrado:

Años de experiencia en unidad renal:

Actualmente enfermero de: hemodiálisis: peritoneal:

6. Con respecto al tratamiento dialítico, la terapia que preserva mejor la FRR es:

a. Hemodiálisis.

b. Diálisis peritoneal continua ambulatoria.

c. Diálisis peritoneal automatizada.

d. Hemodiafiltración.

7. A continuación encontrará cuidados de enfermería para preservar la FRR; escribir en los paréntesis la letra correspondiente al programa: hemodiálisis $(H)$, diálisis peritoneal (P), si alguno(s) corresponde(n) a los dos tratamientos escribir (D)

a. Valorar el peso seco ( ).

b. Controlar el peso prediálisis ( ).

c. Control adecuado de la ultrafiltración ( ).

d. Controlar la TA ( ).

e. Contabilizar la orina cada dos meses ( ).

f. Evitar la hipotensión arterial ( ).

g. Valorar edemas e ingurgitación yugular ( ).

h. Educar al paciente sobre control de la ingesta de sal y líquidos ( ).

i. Control de bicarbonato del líquido de diálisis ( ).

j. Verificar el consumo de proteínas ( ).

k. Evitar los cambios bruscos de volemia en el paciente ( ).

1. Hacer consciente la gravedad de la automedicación ( ).

m. Verificar los reportes de laboratorio de creatinina y BUN ( ).

\section{Los fármacos que deterioran la FRR en mayor grado son:}

a. AINES y aminoglucósidos.

b. Corticoides y IECAS.

c. Agentes yodados y corticoides.

d. IECA y AINES.

\section{9. ¿Cuál de las siguientes patologías causa mayor pérdida de la FRR?}

a. Nefropatía por analgésico.

b. Nefropatía por mieloma.

c. Nefropatía isquémica.

d. Glomerulonefritis rápidamente progresiva. 
nefrólogo y dos enfermeros). Los criterios de selección de los expertos fueron: médicos nefrólogos o enfermeros con experiencia en UR y/o docencia en el área mayor a tres años. Debían definir si los dominios eran suficientes o sugerir si se requerían otros, evaluar el nombre, agregar o eliminar preguntas (ítem) a los diferentes dominios que consideraran pertinentes, corregir redacción y estilo, y dar sugerencias. Los expertos evaluaron el cuestionario de manera independiente dando unas primeras observaciones y correcciones que fueron registradas en un formato de recolección de datos. Con la información obtenida se realizaron modificaciones al instrumento, teniendo como producto una segunda versión, la cual fue entregada para revisión a cinco expertos (dos nefrólogos y tres enfermeras), siguiendo la misma metodología. El cuestionario resultante consta de 25 preguntas en cuatro dominios: conocimientos técnico-científicos de la FRR, conocimientos sobre la importancia de FRR en los diferentes sistemas, conocimientos sobre los factores que preservan la FRR y conocimientos sobre factores que deterioran la FRR. Se diseñó para ser autoadministrado y no contiene preguntas abiertas. Los investigadores y expertos orientaron el diseño de las preguntas con un nivel bajo de complejidad, identificando los elementos mínimos que deben hacer parte de los conocimientos teóricos de los enfermeros que laboran en programa de diálisis. La Figura 1 resume la elaboración del cuestionario.

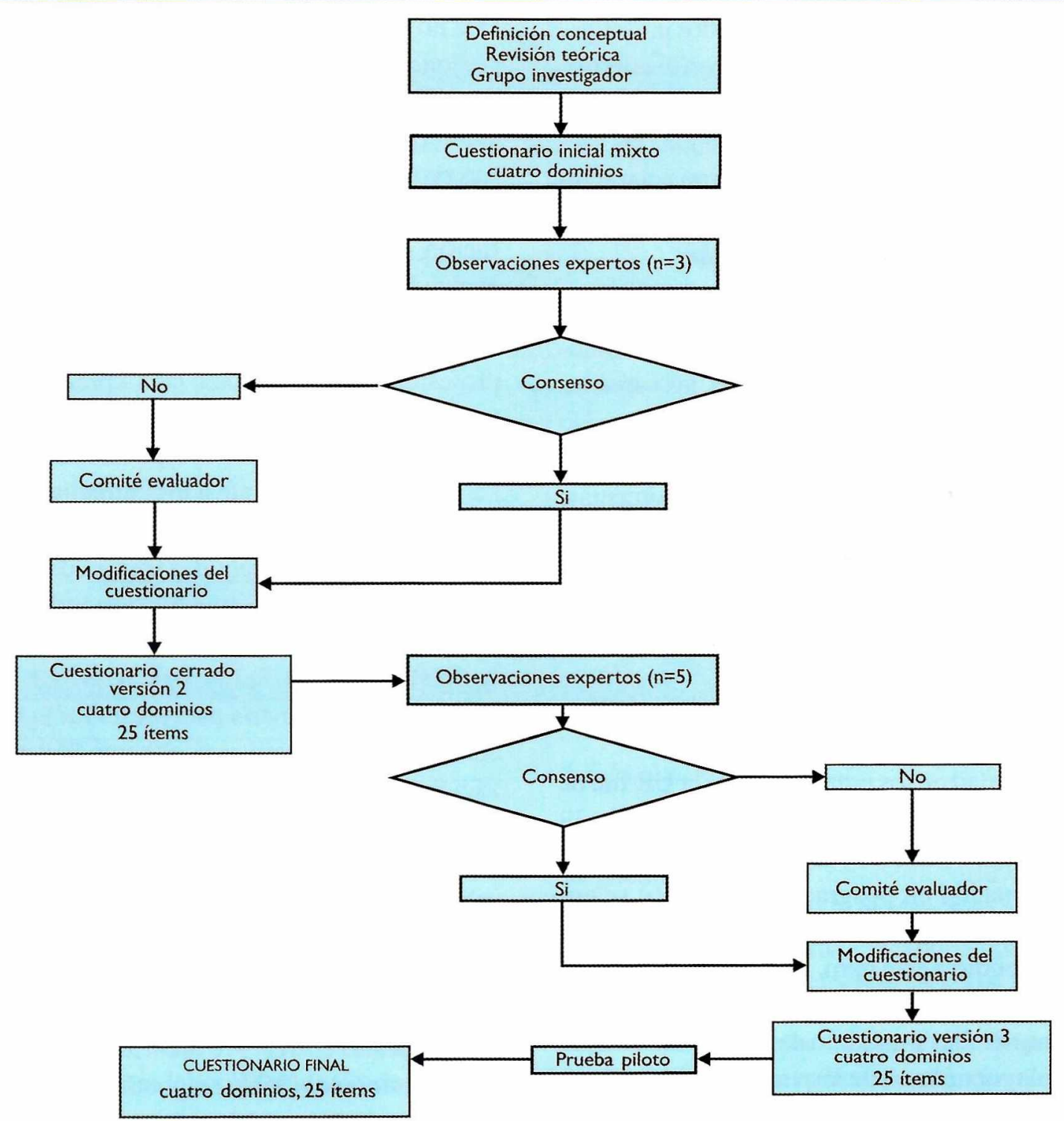

Figura I. Proceso para elaboración de cuestionario. 


\section{Fase 2: Aplicación del cuestionario}

Objetivo: describir los conocimientos teóricos sobre la FRR de los profesionales de enfermería que laboran en una unidad renal de Bogotá. Metodología: estudio aprobado por el comité de investigaciones de la Facultad de Enfermería de la Fundación Universitaria de Ciencias de la Salud. Los profesionales dieron su consentimiento por escrito previo al diligenciamiento del cuestionario. Se realizó un estudio descriptivo de corte transversal.El cuestionario se aplicó a un total de once enfermeros con experiencia mayor a un año ya fuera en hemodiálisis o diálisis peritoneal, vinculados a la misma unidad renal. No se incluyeron enfermeros temporales que estuvieran cubriendo vacaciones o licencias. La recolección de los datos fue supervisada en forma directa por los investigadores en la unidad renal, quienes entregaron el cuestionario a los profesionales de los diferentes turnos en el mismo día. El tiempo máximo para responder las preguntas se definió en 30 minutos. Se hicieron aclaraciones para evitar tachones o enmendaduras en las respuestas.

Análisis estadístico: se utilizó la estadística descriptiva para presentar la información. Las variables cuantitativas se resumen con el promedio o mediana, las variables cualitativas se presentan en forma de frecuencias. No se define un punto de corte para expresar el nivel de conocimiento. Los resultados se expresan en porcentaje de respuestas correctas e incorrectas. La tabulación de los datos se realizó con el programa Microsoft Office Excel versión 2003.

\section{Resultados}

El promedio de edad de los enfermeros de la UR fue de 31.6 años con una mínima de 25 y una máxima de 39 años, el $82 \%$ (9) son mujeres y un $18 \%$ (2) hombres. El $64 \%$ tiene formación en posgrado. El 55\% (6) se encuentra en área de hemodiálisis 9\% (2) en CAPD y $36 \%$ (4) que no respondió a este ítem. El tiempo mediano de experiencia laboral en UR fue de cinco años, con un tiempo mínimo de uno y máximo de siete años. Los que presentaron mayor número de respuestas correctas (mayor o igual a 20) tienen edad promedio de 34,6 años, las tres son mujeres, dos de ellas con posgrado y los años de experiencia laboral en promedio son cinco (mediana seis años). En contraste los cuatro profesionales con mayor número de respuestas incorrectas (mayor o igual a diez) tienen edad promedio de 29,5 años, las cuatro son mujeres, dos tienen estudio de posgrado y el promedio de años de experiencia laboral es de 3,25 (mediana tres años). Los resultados obtenidos a partir de las respuestas consignadas en el cuestionario se describen en la Tabla 1.

El dominio con mayor frecuencia de respuestas incorrectas fue el tecnicocientífico conformado por tres ítems: ninguno respondióbien las tres. El 45,4\% (5) contestó bien los ítems 1 y 3 . Sólo dos respondieron correcto el ítem 2 pero estuvieron incorrectas las preguntas 1 y 3 . Dos no contestaron la 1 . Con respecto al dominio importancia de la FRR en los diferentes sistemas, se identificó que cuatro enfermeros (36\%) respondieron las cinco preguntas de esta categoría en forma correcta. Las preguntas con menor frecuencia de respuesta acertada fueron la 5a y $5 \mathrm{~d}$. Las otras preguntas del dominio fueron respondidas correctamente por el $71 \%$ de los evaluados (pregunta 4) y $91 \%$ (preguntas 5 b y $5 \mathrm{c}$ ).

En el dominio de la preservación de la FRR ningún profesional respondió bien las 14 preguntas de esta categoría. La pregunta 6 sobre la terapia que preserva mejor la FRR reporta sus respuestas correctas (55\%). La pregunta 7, referente a los cuidados de enfermería para preservar la función renal residual, el 72\% identificaron los cuidados según la técnica de diálisis, contra $26 \%$ incorrecto y $2 \%$ que no respondieron. Las preguntas con mayor frecuencia de respuesta incorrecta fueron la 7c (82\%), 7e (64\%) y 7k (87\%). Exploran los cuidados de enfermería para preservar la FRR: control adecuado de la ultrafiltración (hemodiálisis), contabilización de orina cada dos meses (ambas terapias) y evitar cambios bruscos de volemia en el paciente (hemodiálisis). Sobre los factores que deterioran laFRR cabe resaltar que la mayoría de los enfermeros (73\%) conoce los medicamentos nefrotóxicos. Con relación a la pregunta 9, sobre las patologías que causan mayor pérdida de la FRR no se presentó mayor diferencia entre las respuestas correctas e incorrectas (55 y 45\%) Cuatro profesionales $(36 \%)$ respondieron bien las dos preguntas de esta categoría. 
Tabla 1. Conocimientos teóricos del profesional de enfermería sobre la FRR

Pregunta

Correcto

(\%)
Incorrecto

(\%)
No responde

(\%)

Conocimientos tecnicocientíîcos de la FRR

\begin{tabular}{|c|c|c|c|}
\hline Pregunta 1 & 73 & 9 & 18 \\
\hline Pregunta 2 & 18 & 82 & 0 \\
\hline Pregunta 3 & 55 & 45 & 0 \\
\hline
\end{tabular}

Conocimientos sobre la importancia de la FRR en los diferentes sistemas

\begin{tabular}{|l|c|c|c|}
\hline Pregunta 4 & 73 & 27 & 0 \\
\hline Pregunta 5. a & 55 & 45 & 0 \\
\hline Pregunta 5. b & 91 & 9 & 0 \\
\hline Pregunta 5. c & 91 & 9 & 0 \\
\hline Pregunta 5. d & 64 & 36 & 0 \\
\hline Pregunta 5. e & 91 & 9 & 0 \\
\hline
\end{tabular}

Conocimientos sobre los factores que preservan la FRR

\begin{tabular}{|c|c|c|c|}
\hline Pregunta 6 & 55 & 45 & 0 \\
\hline Pregunta 7. b & 82 & 18 & 0 \\
\hline Pregunta 7. d & 100 & 0 & 0 \\
\hline Pregunta 7. e & 36 & 45 & 19 \\
\hline Pregunta 7. $\mathrm{h}$ & 91 & 9 & 0 \\
\hline Pregunta 7. i & 91 & 9 & 0 \\
\hline Pregunta $7 . \mathrm{j}$ & 64 & 3 & 0 \\
\hline Pregunta $7 . \mathrm{k}$ & 27 & 73 & 0 \\
\hline Pregunta 7.1 & 100 & 0 & 0 \\
\hline \multicolumn{4}{|c|}{ Conocimientos sobre factores que deterioran la FRR } \\
\hline Pregunta 8 & 73 & 27 & 0 \\
\hline Pregunta 9 & 55 & 45 & 0 \\
\hline
\end{tabular}

\section{Discusión}

Los que presentaron mayor número de respuestas correctas tienen edad promedio de 34,6 años, frente a 29,5 años en el caso de las incorrectas. Entre los enfermeros con mayor número de respuestas correctas el tiempo mediano de experiencia laboral fue de cinco años, en contraste con tres para quienes tuvieron mayores respuestas incorrectas. El reducido tamaño de la muestra no permite sacar conclusiones a partir de estos resultados. Se podría plantear la hipótesis de que el mayor número de años de experiencia laboral contribuye a fortalecer los elementos conceptuales sobre la función renal.

El dominio con menor frecuencia de respuestas acertadas fue el tecnicocientífico, donde se evalúa la determinación de la FRR con recolección y medición de la orina interdialítica. La apropiación de este concepto es funda- 
mental para el cuidado de enfermería y en el ejercicio profesional es deseable que se considere como un elemento de conocimiento obligado.

Es claro que esta investigación no pretendemedir el conocimiento de los profesionales de enfermería sobre la FRR y reconoce que sólo explora un aspecto de las competencias profesionales: "conocer". Pero el hecho de identificar que uno de los profesionales (9\%) que trabaja en la UR no tiene claro el concepto de FRR y que el $18 \%$ (2) no responde a esta pregunta, genera un cuestionamiento que debe resolverse desde la perspectiva de evaluación de competencias para la práctica de enfermería como lo propone la Organización Panamericana de la Salud en el documento de plan estratégico 2005-2007, en el cual se invita a la conformación de un grupo latinoamericanoque se dedique al estudio de las competencias mínimas de egreso y su correspondencia con las competencias ocupacionales o competencias para la práctica.

Difícil explicar cómo algunos graduados que pueden tener o no un ejercicio profesional que responda a las exigencias del mercado laboral puedan alejarse del concepto teórico de la definición de la FRR, cuando la preservación de ésta constituye en elemento prioritario de su labor cotidiana. De otra parte, el dominio donde se observó mayor conocimiento fue el de la importancia de preservar la FRR, lo cual puede reflejar las fortalezas de la formación del enfermero en aspectos relacionados con la prevención. Los principios que rigen la práctica profesional en enfermería deben garantizar cuidados de calidad y actualizados a quienes reciben sus servicios y desde la perspectiva de cuidado se pretende mantener la salud o evitar el deterioro. En este dominio las preguntas con menor frecuencia de respuesta acertada fueron: "la pérdida de la FRR aumenta el riesgo de presentar malnutrición (pregunta 5a) y anemia (pregunta 5d)". El desconocer que la preservación de la FRR facilita la acción de la eritropoyetina y por ende el control de la anemia, puede generar descuidos en la práctica diaria del cuidado del paciente en diálisis que podrían conducir a la omisión de algunas recomendaciones orientadas a mantener la FRR el mayor tiempo posible.

Las preguntas que tuvieron $100 \%$ de respuestas correctas (7d y 7l) hacen referencia a los cuidados de enfermería para preservar la FRR en cuanto al control de la presión arterial y la gravedad de la automedicación. Esto se podría explicar como se dijo antes por la formación en prevención, pero además por la amplia divulgación en medios tanto profesionales o no de estos conceptos que son de manejo público aún en ámbitos externos a la salud. Sería inaceptable que alguno de los profesionales no hubiera respondido de manera correcta estas preguntas. Aquellas con mayor frecuencia de respuesta incorrecta 7c (82\%), 7e (64\%) y 7k (87\%) exploran los cuidados de enfermería para preservar la FRR. Los elementos que evalúan son: control adecuado de la ultrafiltración (hemodiálisis), contabilización de orina cada dos meses (ambas terapias) y evitar los cambios bruscos de volemia en el paciente (hemodiálisis). Para esta última pregunta no sería, desde la óptica del mínimo desempeño, aceptar que un profesional no tenga claridad sobre los efectos de los cambios de la volemia en la preservación de nefronas que son responsables de la diuresis residual.

Es importante anotar que el diseño del cuestionario validado por cinco expertos puede sobreestimar las respuestas correctas para las preguntas con dos opciones. Es probable que si se hubieran presentado con múltiples respuesta y selección única, la posibilidad de incorrectas fuera mayor. Si bien este trabajo explora elementos teóricos, no puede desconocer la relación teórico-práctica presente en el ejercicio de la enfermería y rescata la perspectiva de integralidad en el abordaje de los pacientes. También hay que reconocer que en el proceso de conceptualización y adquisición de conocimiento teórico entendido como “el saber qué", no siempre se correlaciona con el conocimiento práctico identificado por "el saber como". ${ }^{5}$

El interés en explorar los conceptos teóricos de los enfermeros acerca de la preservación de la FRR parte del supuesto de que el conocimiento teórico basado en herramientas conceptuales es un proceso facilitador para el desarrollo del proceso del cuidado de enfermería, aunque no siempre está relacionado con elementos prácticos. Es decir, un profesional puede revelar algunos vacíos de conocimientos en los que se consideran los elementos conceptuales fundamentales, pero tener una práctica profesional adecuada. Sin embargo, siempre será convenientereforzar elementos teóricos.

Conclusión: este trabajo permite hacer una primera aproximación a la identificación de conceptos teóricos 
que manejan los profesionales de enfermería que trabajan en UR. Los resultados muestran que hay necesidad de revisar algunos y se propone desarrollar una actividad académica para fortalecer elementos teóricos que pudieran contribuir a brindar un mejorcuidado de enfermería a los pacientes en terapia dialítica. Se requieren de estudios futuros para evaluar si existe relación entre el conocimiento de la FRR con la práctica que ejerce el enfermero para preservarla.

\section{Referencias}

1. López-Viñas C, Jaramillo AC, Jaimes J, Muñoz JE, Murcia B, Sánchez DC. Prevalencia de enfermedad renal y entidades asociadas: una base para la promoción de la salud en la población de Simijaca. Rev Fac Med. 2005; 10(1): 63-70.

2. Rodríguez Benítez P, Gómez Campderá FJ. Importancia de la función renal residual en pacientes en hemodiálisis. Nefrología [serie en Internet]. 2002 [citado Jul 2006]; 22(2): [aprox. 6 p.]. Disponible en: URL: http://www.revistanefrologia.com/mostrarfile. asp?ID=2471
3. Wang YM, Lai KN. The importance of residual renal function in dialysis patients. Kidney Int.. 2006; 69(10): 1726-32.

4. Churchill D, Taylor W, Keshaviah P. Adecuancy of dialysis and nutrition in continuous peritoneal dialysis: association with clinical outcomes. Canada-USA (CANUSA) Peritoneal Dialysis Study Group. J Am Soc Nephrol. 1996 Feb; 7(2): 198-207.

5. Ramirez B, Pulido Gomez M, Roncal D, Lobo MT, Albiach R, Rodriguez Benitez P. Función renal residual. Su importancia en el manejo del paciente en diálisis. Rev Soc Esp Enferm Nefrol [serie en Internet]. 2001 [citado Jul 2006]; 4(1): [aprox. 6 p]. Disponible en: URL: http://www.seden.org/files/rev22_1.pdf

6. Por la cual se reglamenta la profesión de enfermería en Colombia. Ley 266/1996 de 25 de enero. Diario Oficial No. 42.710

7. Por la cual se dictan disposiciones en materia de responsabilidad deontológica para el ejercicio de la profesión de enfermería en Colombia se establece el régimen disciplinario correspondiente y se dictan otras disposiciones. Ley 911/2007 de octubre de 2005. Diario Oficial No 45.693, (6-10-2004).

8. Gómez, E. Monterrosas, A. Desafíos para la formación de técnicos en salud: construcción de competencias educacionales. Washington: OPS/OMS; 1999. 